\title{
Flash Sintering of Commercial Zirconium Nitride Powders
}

Diego Dubois ${ }^{1}$, Amirali Eskandariyun ${ }^{1}$,Suprabha Das ${ }^{1}$, Andriy Durygin ${ }^{1}$, and Zhe Cheng ${ }^{1}$

${ }^{1}$ Department of Mechanical and Materials Engineering

Keywords: flash sintering, zirconium nitride, ceramics

Flash sintering is an electrical field-assisted densification technique that requires passing a current through a ceramic powder compact. Pressure-assisted flash sintering of commercially available Zirconium Nitride ( $\mathrm{rrN}$ ) powders has been demonstrated. Near fully dense samples can be obtained within a short period of time. The influences of parameters such as electrical field strength, voltage ramping rate, current limit, external pressure, pre-heating, and holding time on the onset of the flash event were investigated. Some post-flash sintered samples were subjected to the same condition to observe if the material would experience repeated flash. In addition, material properties such as density and hardness were measured and correlated with SEM and XRD. Implications of the observations on underlying flash sintering mechanism will also be discussed. 